\title{
Effect of guided imagery training on death anxiety of nurses at COVID-19 intensive care units: a quasi-experimental study
}

\author{
Sedigheh Sanadgol', Mohammadreza Firouzkouhi'1, Mahin Badakhsh², \\ Abdolghani Abdollahimohammad' ${ }^{1}$, Aziz Shahraki-vahed ${ }^{1}$ \\ ${ }^{1}$ Department of Nursing, School of Nursing and Midwifery, Zabol University of Medical Sciences, Zabol, Iran \\ ${ }^{2}$ Department of Midwifery, School of Nursing and Midwifery, Zabol University of Medical Sciences, Zabol, Iran \\ Neuropsychiatria i Neuropsychologia 2020; 15, 3-4: 83-88
}

Address for correspondence:

Abdolghani Abdollahimohammad, MD

Department of Nursing

School of Nursing and Midwifery

Zabol University of Medical Sciences

Zabol, Iran

e-mail: abdalqani@gmail.com

\begin{abstract}
Introduction: Death anxiety (DA) is one of the stresses experienced by intensive care unit (ICU) nurses on a daily basis. Failure to pay attention to DA causes psychological problems and job dissatisfaction for nurses and failure to receive proper and adequate care for patients.

Aim of the study: To determine the effect of guided imagery (GI) on DA among ICU nurses.

Material and methods: This quasi-experimental (pre-test-post-test) study was conducted on 50 ICU nurses in one educational hospital of Zabol University of Medical Sciences in south east Iran from 10 January to 10 June 2020. The participants were selected using the convenience sampling method then divided into two groups, intervention $(n=25)$ and control $(n=25)$. In the intervention group, participants received GI using theory education by lecture, pamphlet, booklet, and practice. In the control group participants received no specific intervention. In both groups DA was assessed by the researcher using the Templer DA scale at the start of study, and one month after intervention. SPSS ver. 23 was used for data analysis.

Results: The results showed in the intervention group after one months of GI mean score of DA significantly decreased from 53.28 to $43.48(p=0.01)$. In the control group there was no significant difference before and after intervention $(p>0.05)$.

Conclusions: Based on the results of the present study GI can be used as an effective method to decrease the DA and improve the mental health of nurses in ICU.
\end{abstract}

Key words: imagery, psychotherapy, mental health, anxiety, nurses.

\section{Introduction}

Coronavirus disease 2019 (COVID-19) has been declared a public health emergency by the World Health Organization. This emerging disease, first seen in China in late 2019, has spread around the world in less than a few months. According to the latest statistics on COVID-19 on October 11, 2020, more than $37,505,587$ people had been infected and more than 1,077,983 people had died.

Various health personnel are involved in the fight against COVID-19. Nurses are at the forefront of the fight against COVID-19 as people who are in direct contact with patients. There is no definitive cure for this disease and it is spread through airborne droplets. The only way to prevent the infection is physical distanc- ing and avoiding contact with infected patients. Therefore, due to frequent visits to hospitals, healthcare personnel, especially intensive care unit (ICU) nurses who are in 24-hour contact with critically ill COVID-19 patients, are under the most psychological and physical stress. ICU nurses experience more death anxiety (DA) due to daily exposure to patient mortality (Moudi et al. 2017). Nurses are more likely to experience a variety of psychological symptoms, including DA, due to their daily encounters with COVID-19 patients, especially considering the lack of protective facilities. Studies have shown that more than $74 \%$ of nurses suffer from DA (Moudi et al. 2017).

Death anxiety is defined as an unusual and great fear of death combined with feelings of 
fear of death or apprehension when thinking about the process of dying or what happens after death (Supplementary Medicine 2012). Caring for critically ill and dying patients is a difficult aspect of the nursing profession (Parsaie and Mohamadi 1999). Nurses with high DA avoid talking about death with clients, while dying people often like to talk about it with caregivers and relatives (Shorofi and Karimzadeh 2015). Death anxiety can lead to complications in nurses such as burnout, decreased efficiency, decreased patient satisfaction, leaving the profession, incompatibility with colleagues, depression and even suicide, and it affects the quality of care (Woo et al. 2013; Ranjbar et al. 2017). Therefore, we can improve the quality and effectiveness of care, ensure more effective presence of ICU nurses and their greater satisfaction with the work environment through timely diagnosis and effective treatment of DA (Ranjbar et al. 2017). There are several methods to reduce anxiety and the ability to cope with stress, including pharmacological and non-pharmacological methods. In addition to the possibility of drug dependence, the pharmacological method is associated with other side effects such as cardiac dysrhythmias and death. Non-pharmacological methods are non-invasive and often have no side effects. One of these methods is guided imagery (GI) (Masoumi et al. 2016). Previous studies have shown that GI improves the quality of life of patients undergoing coronary artery bypass graft surgery (Jacobson 1929), reduces negative and dysfunctional thoughts and attitudes in women with breast cancer (Bahrami-Eyvanekey et al. 2017), reduces the severity of depression and increases hope among dialysis patients (Sadeghi et al. 2017). It reduces systolic and diastolic blood pressure during pregnancy (Ali Far and Nokani 2013), and reduces anxiety and promotes general health of the community (Azimian et al. 2017), and has been studied in different groups. In GI mental imagery, one replaces disturbing memories with positive imagery. It is an easy and safe method, is known to have no side effects and can be used at any time and place. Therefore, it can be implemented in any environment, even in a hospital environment, without disturbing the process of patient care (Gholami et al. 2015).

Nowadays, considering the vital role of and need for nurses in the management of COVID-19 patients, the prolongation of the disease course, the daily increase of patients and critically ill patients, the increase of DA, its impact on the quality of care provided, and the need to use effective approaches to reduce DA without possible complications, the present study was performed with the aim of investigating the effect of GI training on DA ICU nurses.

\section{Material and methods}

\section{Design}

This quasi-experimental (pre-test-post-test) study was performed on 50 ICU nurses of a teaching hospital affiliated to Zabol University of Medical Sciences from 10 January to 10 June 2020 in southeastern Iran. Participants were selected using convenience sampling. Participants were then randomly assigned to the intervention and control groups. Inclusion criteria included having a bachelor's degree in nursing and higher, at least one year of experience in ICUs, no history of known mental illness, no use of psychotropic drugs in the last six months, and no experience of GI as an anxiety management method before entering the study (Sadeghi et al. 2017). Exclusion criteria also included inaccuracy in completing the questionnaire, transfer and death of the nurse. The study sample size was calculated to be 50 ICU nurses ( $n=25$ per group) based on the study of Aghajani et al. (2010; Dadgari et al. 2015), the following statistical formula:

$$
\mathrm{N}=2 \mathrm{c} \times(\operatorname{vsd} 12+\mathrm{sd} 22 / \mathrm{m} 1-\mathrm{m} 2)^{2}
$$

taking into account $20 \%$ sample drop-out, $95 \%$ confidence interval, and test power $=80 \%$.

\section{Instruments}

Data collection instruments included two self-report questionnaires. The first questionnaire contained demographic questions, including age, sex, and marital status, level of education, employment status, shift work, and work experience. The second questionnaire was the 15-item Templer DA scale (Sadeghi et al. 2017). Each question was answered based on a 5-point Likert scale ranging from strongly disagree (Worldometers 2020) to strongly agree (Parsaie and Mohamadi 1999). A higher score indicates more DA. The validity and reliability of the instrument in Iran were confirmed by Sharif Nia et al. with Cronbach's $\alpha$ of 0.91 .

\section{Data collection}

After coordination with ICU authorities of the hospital, the objectives of the study were explained to the nurses and eligible participants voluntarily participated in the study. Participants were divided into control and intervention groups through drawing lots. The questionnaire was completed 
by all participants before the intervention. Then, patients of the intervention group received training in GI in 5-member groups for 90 minutes.

In the first session, the objectives of the research, DA, and its causes, complications and treatments were explained with the emphasis on GI. In the second session, the GI implementation procedure was taught in a practical way and then each person performed it before the researcher. To this end, the subjects were asked to close their eyes and inhale deeply through their nose and exhale deeply through their mouth. They were then asked to remember one of the painful experiences of dying and freely express the subsequent feelings (fear, sadness, guilt, etc.). They were also requested to remove those emotions from their body through imagination, transfer them to a balloon, and release them so that these emotions would go away from them. They then slowly opened their eyes and recalled that memory and re-examined the feelings that resulted from that experience. If those memories were still disturbing, they continued the GI process until those feelings disappeared. It is recommended to take at least three sessions a week at home, preferably before going to bed, to eliminate anxiety risk factors. The control group received no intervention during this period. One month later, the Templer DA scale was completed again by individuals in both intervention and control groups.

\section{Ethical considerations}

This research was approved by the Ethics Committee of Zabol University of Medical Sciences (Ethics code: IR.ZBMU.REC.1398.113) Prior to the study, the objectives of the study were fully explained to the participants and everyone was assured that they could withdraw from the study at any time. Oral and written consent was obtained from the participants. All participants were free to withdraw from the study at any time.

\section{Data analysis}

Data analysis was carried out using descriptive statistics (relative frequency, mean and standard deviation) and inferential statistics of $\chi^{2}$ and independent $t$-test and analysis of variance (ANOVA) in SPSS ver. 23.

\section{Results}

A total of 50 ICU nurses participated in the present study. Table 1 shows that most nurses were female, married, and had a bachelor's degree. The mean age of the intervention and control groups was 33.56 and 31.24 years, respectively. Most of the samples in the intervention and control groups had 8 and 10.08 year of work experience, respectively. There was no statistically significant difference between the two groups in terms of the frequency of qualitative variables and the mean of quantitative variables.

Independent $t$-test showed no statistically significant difference between intervention and control groups in terms of pre-intervention DA scores $(p>0.05)$. However, there was a statistically significant difference between the two groups in terms of mean DA scores after the intervention $(p=0.004)$. Also the ANOVA test showed a statistically significant difference between the intervention and control groups in term of the mean DA score by controlling the pre-test variable $(p=0.001)$ (Table 2$)$.

\section{Discussion}

The aim of the present study was to determine the effect of GI on DA of ICU nurses. The findings showed a significant decrease in DA level among ICU nurses of the intervention group compared to the control group after the intervention.

Although there has been no study demonstrating the effectiveness of GI on DA, many studies have shown its effectiveness on other physical and mental problems such as pain, insomnia, nausea and vomiting, stress, anxiety, and depression in different groups. Guided imagery reduces anxiety in surgical patients (Aghajani et al. 2010), multicultural groups (Felix et al.

Table 1. Demographic characteristics of participants

\begin{tabular}{llcc} 
Variable & male & $\begin{array}{c}\text { Intervention } \\
n(\%)\end{array}$ & $\begin{array}{c}\text { Control } \\
n(\%)\end{array}$ \\
\cline { 2 - 4 } Gender & female & $15(40)$ & $12(48)$ \\
\hline Marital status & married & $18(72)$ & $13(52)$ \\
\cline { 2 - 4 } & single & $7(28)$ & $2(8)$ \\
\hline Education & bachelor & $21(84)$ & $20(80)$ \\
\cline { 2 - 4 } level & master & $4(16)$ & $5(20)$ \\
\hline Shift type & fixed & $13(52)$ & $12(48)$ \\
\cline { 2 - 4 } & rotating & $12(48)$ & $13(52)$ \\
\hline Age (years) & mean (SD) & $33.56(5.57)$ & $31.24(5.21)$ \\
\hline Job experience & mean (SD) & $8.00(5.27)$ & $10.08(5.78)$ \\
\hline
\end{tabular}


Table 2. Comparison of mean and standard deviation of death anxiety in the two groups of intervention and control before and after the intervention

\begin{tabular}{lccc} 
Stage of study & \multicolumn{3}{c}{ Death anxiety } \\
\cline { 2 - 4 } & $\begin{array}{c}\text { Intervention }(n=25) \\
\text { Mean } \pm \text { SD }\end{array}$ & $\begin{array}{c}\text { Control }(n=25) \\
\text { Mean } \pm \text { SD }\end{array}$ & P-value \\
Before intervention & $53.28(7.54)$ & $50.84(7.63)$ & 0.261 \\
\hline After intervention & $43.48(6.13)$ & $49.48(7.90)$ & 0.004 \\
\hline \multirow{2}{*}{ Mean $(95 \% \mathrm{Cl})$} & 0.001 \\
\cline { 2 - 3 } & \multicolumn{2}{c}{ Intervention } & \\
\hline
\end{tabular}

2018), pregnant women (Nguyen and Brymer 2018; Nasiri et al. 2018), breast cancer patients (Jallo et al. 2014), health professionals (Chen et al. 2015), and joint replacement patients (Rao and Kemper 2017). Guided imagery has also reduced stress levels in pregnant women (Nguyen and Brymer 2018; Nasiri et al. 2018), breast cancer patients (Jallo et al. 2014), health professionals (Chen et al. 2015), fibromyalgia patients (Forward et al. 2015), and thyroid cancer patients (Menzies et al. 2014). Additionally, it has reduced depression in pregnant women (Nasiri et al. 2018), breast cancer patients (Jallo et al. 2014), and fibromyalgia patients (Forward et al. 2015). Guided imagery has also reduced fatigue in African-American pregnant women (Jallo et al. 2014), women with fibromyalgia (Forward et al. 2015), and multiple sclerosis patients (Lee et al. 2013). Other physical symptoms, including pain (Forward et al. 2015; Rao and Kemper 2017), insomnia (Jallo et al. 2014), and nausea and vomiting in pregnant women (Case et al. 2018), also decreased following GI intervention. Furthermore, preoperative cortisol levels decreased in patients after GI intervention (Aghajani et al. 2010) and after complete knee replacement (Shakiba et al. 2019).

Although the mechanism of action of mindbody interventions, such as GI, is not known precisely, some studies (Jacobson et al. 2016; Lewandowski et al. 2011; Haruna et al. 2019) have shown that mental imagery alleviates symptoms by activating the hypothalamic-pituitary-adrenal axis, balancing sympathetic and parasympathetic activity, altering perceptual processes, and affecting the mind at a deeper or subconscious level. A literature review did not reveal any study that demonstrated the effectiveness of GI on DA. However, previous studies have shown the effectiveness of GI on the physical and psychological aspects of human beings, which is consistent with the present study. Mental imagery reduces anxiety in surgical patients (Aghajani et al. 2010), multicultural groups (Felix et al. 2018), pregnant women (Nasiri et al. 2018; Nguyen et al. 2018), breast cancer patients (Jallo et al. 2014), health professionals (Chen et al. 2015), and joint replacement patients (Rao and Kemper 2017). Guided imagery has also reduced stress levels in pregnant women (Nasiri et al. 2018; Nguyen et al. 2018), breast cancer patients (Jallo et al. 2014), health professionals (Chen et al. 2015), fibromyalgia patients (Forward et al. 2015), and thyroid cancer patients (Menzies et al. 2014). In addition, it has reduced depression in pregnant women (Jallo et al. 2014), breast cancer patients (Jallo et al. 2014), and fibromyalgia patients (Forward et al. 2015). Guided imagery also reduced fatigue in African-American pregnant women (Nasiri et al. 2018), women with fibromyalgia (Forward et al. 2015), and multiple sclerosis patients (Lee et al. 2013). Other physical symptoms, including pain (Forward et al. 2015; Rao and Kemper 2017), insomnia (Jallo et al. 2014), and nausea and vomiting in pregnant women (Case et al. 2018) also decreased following GI intervention. Guided imagery also improved the self-efficacy (Forward et al. 2015; Chen et al. 2015), quality of life (Shakiba et al. 2019; Lee et al. 2013), mood (Lee et al. 2013), and empathy (Chen et al. 2015) of the participants in these studies. Also, a review study by Menzies et al. (2014; Haruna et al. 2019) shows that cognitive-behavioral therapy has led to a greater reduction in DA compared to other treatments. However, none of the studies used GI intervention. All of these studies support the results of the present study on the effectiveness of GI in reducing DA among ICU nurses.

However, the findings of the present study were inconsistent with the findings of Nilsson et al. The study by Kwekkeboom and Bratzke (2016) that showed GI intervention was not 
effective in reducing pain and discomfort in children during vaccination, which may be due to the differences between invasive and noninvasive methods, acute and chronic disease, as well as the imagination of children and adults.

Most mental imagery studies focus on teaching relaxation processes. In the present study, participants were recommended to be in a comfortable position and then do mental imagery to release situations that cause DA in them. Although no audio or video file was used to guide the process in this method, the GI intervention steps were performed in writing and also in a form of practical training in the presence of the researcher (Nilsson et al. 2015).

One of the strengths of the present study is to determine the effect of GI intervention on DA in people who face it on a daily basis and more frequently than other people in society. However, the present study has some limitations. Training and relaxation can reduce anxiety, but they are an integral part of mental imagery and it was not possible to control their effect in the present study. This study was performed under normal and non-critical conditions, so its results cannot be generalized to critical conditions such as the COVID-19 pandemic. On the other hand, the present study was performed only on ICU nurses of one hospital, so the results can be generalized only to normal conditions and similar groups.

Although the participants of the current study were in the same religious and cultural conditions, considering the impact of religious and cultural beliefs and life-threatening critical conditions on DA, it is suggested to carry out future studies on different groups.

\section{Conclusions}

Guided imagery is effective in reducing the DA of ICU nurses and can be used as a complementary treatment method to promote mental health and the level of quality of services provided. These techniques may yield effective results among the caregiver population as well as other people who are in a therapeutic relationship with patients, such as social workers, doctors, nurses, hospital staff, and all members of the treatment staff. It is noteworthy that so far there has been no evidence of adverse effects associated with anxiety coping techniques. It is hoped that we will see a better situation regarding the mental health of the nursing personnel and similar groups in the future by using the results of the present study and adopting appropriate methods.

\section{References}

1. Aghajani M, Valiee S, Tol A. Death anxiety amongst nurses in critical care and general wards. Iran J Nurs 2010; 23: 59-68.

2. Ali Far E, Nokani M. Effectiveness of guided visualization and mental imagery in decreasing the symptoms of depression and increasing hopefulness among hemodialysis patients. J Thought Behav Clin Psychol 2013; 7: 81-90.

3. Azimian G, Pashazadeh F, Alipour Heidary M, et al. The effects of progressive muscle relaxation and guided imagery on gestational hypertension. Complement Med J 2017; 7: 1906-1917.

4. Bahrami-Eyvanekey Z, Ramezani-Badr F, Amini K, et al. Comparison of the effect of two guided visualization methods and progressive muscle relaxation on the quality of life of patients undergoing coronary artery bypass graft surgery. Iran J Nurs Res 2017; 12: 7-15.

5. Case LK, Jackson P, Kinkel R, et al. Guided imagery improves mood, fatigue, and quality of life in individuals with multiple sclerosis: an exploratory efficacy trial of healing light guided imagery. J Evid Based Integr Med 2018; 23: $2515690 \times 17748744$.

6. Chen SF, Wang HH, Yang HY, et al. Effect of relaxation with guided imagery on the physical and psychological symptoms of breast cancer patients undergoing chemotherapy. Iran Red Crescent Med J 2015; 17: e31277.

7. COVID-19 Coronavirus Pandemic. Worldometers 2020. Available from: https://www.worldometers.info/coronavirus/.

8. Dadgari F, Rouhi M, Farsi Z. Death anxiety in nurses working in critical care units of AJA hospitals. J Military Caring Sci 2015; 3: 150-157.

9. Felix MMDS, Ferreira MBG, Oliveira LF, et al. Guided imagery relaxation therapy on preoperative anxiety: a randomized clinical trial. Rev Lat Am Enfermagem 2018; 26: e3101.

10. Forward JB, Greuter NE, Crisall SJ, et al. Effect of structured touch and guided imagery for pain and anxiety in elective joint replacement patients - a randomized controlled trial: M-TIJRP. Perm J 2015; 19: 18-28.

11. Gholami Jam F, Kheftan P, Eghlima M, et al. Stress management techniques and its effects on health promotion. Q J Soc Work 2015; 4: 18-27.

12. Haruna M, Matsuzaki M, Ota E, et al. Guided imagery for treating hypertension in pregnancy. Cochrane Database Syst Rev 2019; 4: CD011337.

13. Jacobson E. Progressive relaxation. University of Chicago Press, Chicago 1929.

14. Jacobson AF, Umberger WA, Palmieri PA, et al. Guided imagery for total knee replacement: a randomized, placebo-controlled pilot study. J Altern Complement Med 2016; 22: 563-575.

15. Jallo N, Ruiz RJ, Elswick RK Jr, et al. Guided imagery for stress and symptom management in pregnant african american women. Evid Based Complement Alternat Med 2014; 2014: 840923.

16. Kwekkeboom KL, Bratzke LC. A systematic review of relaxation, meditation, and guided imagery strategies for symptom management in heart failure. J Cardiovasc Nurs 2016; 31: 457-468.

17. Lee MH, Kim DH, Yu HS. The effect of guided imagery on stress and fatigue in patients with thyroid cancer undergoing radioactive iodine therapy. Evid Based Complement Alternat Med 2013; 2013: 130324. 
18. Lewandowski W, Jacobson A, Palmieri PA, et al. Biological mechanisms related to the effectiveness of guided imagery for chronic pain. Biol Res Nurs 2011; 13: 364-375.

19. Masoumi M, Tahmasebi R, Jalali M, et al. The study of the relationship between job stress and spiritual health of nurses working in intensive care ward at Bushehr Hospitals. Nurs J Vulnerable 2016; 3: 37-47.

20. Menzies V, Lyon DE, Elswick RK Jr, et al. Effects of guided imagery on biobehavioral factors in women with fibromyalgia. J Behav Med 2014; 37: 70-80.

21. Moudi S, Bijani A, Tayebi M, et al. Relationship between death anxiety and mental health status among nurses in hospitals affiliated to Babol University of Medical Sciences. J Babol Univ Med Sci 2017; 19: 47-53.

22. Nasiri S, Akbari H, Tagharrobi L, et al. The effect of progressive muscle relaxation and guided imagery on stress, anxiety, and depression of pregnant women referred to health centers. J Educ Health Promot 2018; 7: 41.

23. Nguyen J, Brymer E. Nature-based guided imagery as an intervention for state anxiety. Front Psychol 2018; 9: 1858

24. Nilsson S, Forsner M, Finnström B, et al. Relaxation and guided imagery do not reduce stress, pain and unpleasantness for 11- to 12-year-old girls during vaccinations. Acta Paediatr 2015; 104: 724-729.

25. Parsaie FA, Mohamadi SH. A Study on burnout in emergency wards nurses. Q J Fund Mental Health 1999; 1: 80-85.

26. Ranjbar H, Mojalli M, Namdar Areshtanab H. Job burnout among nurses working in ICUs affiliated to the educational and therapeutic centers of Mashhad University of Medical Sciences. J Torbat Heydariyeh Univ Med Sci 2017; 5: 56-61.

27. Rao N, Kemper KJ. The feasibility and effectiveness of online guided imagery training for health professionals. J Evid Based Complementary Altern Med 2017; 22: 54-58.

28. Sadeghi A, Shiroodi S, Tarkhan Kh, et al. The effect of grammatical mental imagery treatment on processing and processing one's thoughts is negative ineffective worries of women with cancer. J Soc Sci Res 2017; 6 .

29. Shakiba M, Parsi H, Pahlavani Shikhi Z, et al. The effect of psycho-education intervention based on relaxation methods and guided imagery on nausea and vomiting of pregnant women. J Family Reprod Health 2019; 13: 47-55.

30. Sharif Nia H, Pahlevan Sharif S, Goudarzian AH, et al. An evaluation of psychometric properties of the Templer's Death Anxiety Scale-Extended among a sample of Iranian chemical warfare veterans. Hayat 2016; 22: 229-244.

31. Shorofi SA, Karimzadeh M. Factors associated with burnout in nursing staff: a review article. Clin Exc 2015; 3: 56-70.

32. Supplementary Medicine, Nutrition; Deep Breathing Exercises: The Answer To Anxiety, Stress, And Depression. Mental Health Business Week 2012.

33. Woo YW, Kim KH, Kim KS. Death anxiety and terminal care stress among nurses and the relationship to terminal care performance. Kor J Hospice Palliat Care 2013; 16: 33-41. 